OPEN ACCESS

Edited by:

Elisabetta Rovida,

University of Florence, Italy

Reviewed by:

Matteo Lulli,

University of Florence, Italy

Wafik S. El-Deiry,

Warren Alpert Medical School

of Brown University, United States

*Correspondence:

David Z. Qian

qianzh@ohsu.edu

${ }^{\dagger}$ These authors have contributed equally to this work

Specialty section:

This article was submitted to

Signaling,

a section of the journal

Frontiers in Cell and Developmental

Biology

Received: 11 June 2021

Accepted: 06 October 2021

Published: 08 November 2021

Citation:

Geng H, Ko HK, Pittsenbarger J,

Harvey CT, Xue C, Liu Q, Wiens S,

Kachhap SK, Beer TM and Qian DZ (2021) HIF1 and ID1 Interplay Confers Adaptive Survival to HIF1 $\alpha$-Inhibition.

Front. Cell Dev. Biol. 9:724059.

doi: 10.3389/fcell.2021.724059

\title{
HIF1 and ID1 Interplay Confers Adaptive Survival to HIF1 $\alpha$-Inhibition
}

\begin{abstract}
Hao Geng ${ }^{1 \dagger}$, Hyun-Kyung Ko ${ }^{1 \dagger}$, Janet Pittsenbarger ${ }^{1}$, Christopher T. Harvey ${ }^{1}$, Changhui Xue', Qiong Liu' ${ }^{1}$, Sadie Wiens ${ }^{1}$, Sushant K. Kachhap², Tomasz M. Beer ${ }^{1}$ and David Z. Qian ${ }^{1 *}$
\end{abstract}

'Prostate Cancer Research Program, OHSU Knight Cancer Institute, Oregon Health \& Science University, Portland, OR, United States, ${ }^{2}$ Sidney Kimmel Comprehensive Cancer Center, Johns Hopkins University, Baltimore, MD, United States

Hypoxia is a universal pathological feature of solid tumors. Hypoxic tumor cells acquire metastatic and lethal phenotypes primarily through the activities of hypoxia-inducible factor 1 alpha $(H I F 1 \alpha)$. Therefore, HIF1 $\alpha$ is considered as a promising therapeutic target. However, HIF inhibitors have not proven to be effective in clinical testing. The underlying mechanism is unclear. We report that oncogenic protein ID1 is upregulated in hypoxia by HIF1 $\alpha$ shRNA or pharmacological inhibitors. In turn, ID1 supports tumor growth in hypoxia in vitro and in xenografts in vivo, conferring adaptive survival response and resistance. Mechanistically, ID1 proteins interfere HIF1-mediated gene transcription activation, thus ID1 protein degradation is accelerated by HIF1 $\alpha$-dependent mechanisms in hypoxia. Inhibitions of HIF1 $\alpha$ rescues ID1, which compensates the loss of HIF1 $\alpha$ by the upregulation of GLS2 and glutamine metabolism, thereby switching the metabolic dependency of $\mathrm{HIF} 1 \alpha$-inhibited cells from glucose to glutamine.

Keywords: ID1, hypoxia, resistance, HIF1, targeted-treatment

\section{INTRODUCTION}

Solid tumor growth is inevitably accompanied by hypoxia, which activates the master transcription regulator - hypoxia inducible factors (HIFs) by increasing HIF $1 \alpha$ and HIF2 $\alpha$ (Harris, 2002; Giaccia et al., 2004; Bertout et al., 2008; Majmundar et al., 2010; Marignol et al., 2013). Although both HIFs promote oncogenesis, HIF $1 \alpha$ appears to predominant in most types of human cancers (Sowter et al., 2003; Löfstedt et al., 2007; Ratcliffe, 2007; Dang et al., 2008; Keith et al., 2012). Clinically, an increase of HIF1 $\alpha$ level is associated with advanced metastatic disease and/or patient mortality in almost all types of solid tumors (Semenza, 2007, 2009, 2010, 2012). Thus, HIF1 $\alpha$ is considered as a promising therapeutic target, which in theory may improve disease outcome and patient survival (Giaccia et al., 2003; Semenza, 2003; Powis and Kirkpatrick, 2004). A growing number of HIF $1 \alpha$-inhibitory agents, including both chemical inhibitors (topotecan, PX-478, YC1, 2-ME2, BAY87-2243, and digoxin) and antisense oligonucleotides (EZN-2968) have shown encouraging antitumor activities in blocking tumor growth and metastasis in multiple preclinical models (Semenza, 2012). However, the preclinical efficacy has not been recapitulated in clinical trials (clinicaltrials.gov). The mechanisms of resistance are not clear.

Inhibitor of DNA binding 1 (ID1) is an oncogenic protein, promoting cancer survival, proliferation, angiogenesis, and metastasis (Lyden et al., 1999; Perk et al., 2005; Gupta et al., 2007; Lasorella et al., 2014). ID1 protein has a helix-loop-helix domain (HLH), which negatively regulates the activity of HLH transcription factors (TF) by decreasing the TF/DNA-binding 
(Sun et al., 1991; Alani et al., 2001). Interestingly, HIF1 $\alpha$ contains a HLH domain, which is essential for the assembly of HIF1-transcirptional complex and activity (Wang et al., 1995). Currently, the interactions between HIF1 and ID1 are unclear.

In this study, we found that ID1 protein is upregulated in response to HIF1 $\alpha$ inhibition, and ID1 in turns supports HIF1-independent tumor growth in hypoxia and in vivo. Mechanistically, this stems from the negative interplay between HIF $1 \alpha$ and ID1. The consequence is that ID1 is upregulated in response to HIF1 $\alpha$-targeted inhibition, which in turn compensates the loss of HIF1 by promoting metabolic adaptation via glutamine metabolisms.

\section{RESULTS}

\section{ID1 Protein Is Negatively Regulated by Hypoxia}

Hypoxia reduces the efficacy of antitumor treatments. Previously, we found that stable overexpression of ID1 sensitizes prostate cancer cells to docetaxel chemotherapy by overwriting cell cycle checkpoints (Geng et al., 2010). In hypoxic condition, however, the ID1-based chemosensitization effect was significantly diminished (Figure 1A). In parallel, the plasmid-driven ID1 protein was reduced (Figure 1B). This hypoxia-dependent reduction of ID1 protein was present in a variety of cancer cell lines (prostate, liver, and brain) that express detectable levels of endogenous ID1 (Figure 1C). In all these cells, the reduction was at the protein level, as ID1 mRNA remained unchanged or slightly increased (Figure 1D), and ID1 protein was rescued by proteasome inhibitor MG132 (Figures 1E,F). To understand the hypoxia-induced protein degradation, we found that the inhibition of ID1 began when the oxygen concentration dropped below 4\%, and re-oxygenation reinstated ID1 (Figure 1G). We next measured the kinetics of ID1 protein degradation with methods described by us previously (Geng et al., 2012). We found that hypoxia decreased ID1 protein half-life from $\sim 110$ to $\sim 30$ min (Figure 1H).

\section{HIF1 $\alpha$ Accelerates ID1 Protein Degradation}

To understand the mechanism for hypoxia to degrade ID1, we used our established RNAi methods to knockdown (KD) HIF1 $\alpha$, HIF2 $\alpha$, or non-target control (Geng et al., 2011, 2012, 2018). We found that hypoxia reduced ID1 and ID3 among the ID-family proteins (Figure 2A). Inhibiting HIF $\alpha$ rescued and reinstated ID1, but not ID3 (Figure 2A). On the other hand, inhibiting HIF2 $\alpha$ had no effect (Figure 2B), suggesting ID1 protein is negatively affected by HIF $1 \alpha$, but not HIF $2 \alpha$. Further, we treated cancer cells with a sub-toxic dose of a pharmacological HIF inhibitor-digoxin (Zhang et al., 2008). We found that cells treated with digoxin consistently expressed higher levels of ID1 proteins in hypoxia compared to solvent-treated controls (Figure 2C). Most commonly, ID1 protein degradation is mediated by polyubiquitination (Bounpheng et al., 1999; Berse et al., 2004; Trausch-Azar et al., 2004). We found that ID1 polyubiquitination was significantly increased in hypoxia, and $\mathrm{HIF} 1 \alpha$ was required for the increase (Figure 2D). Since HIF1 $\alpha$ lacks the function of polyubiquitination, we determined the E3 ubiquitin ligase responsible for ubiquitination of ID1 in hypoxia. There are two known ID1 E3 ligases, Smurf2 and APC/CDH1 (Lasorella et al., 2006; Kong et al., 2011). We used siRNA to specifically inhibit Smurf2 and CDH1 as well as the HIF1 $\alpha$-E3 ligase VHL. We found that silencing $\mathrm{APC} / \mathrm{CDH} 1$ significantly reinstated ID1 levels in hypoxia (Figure 2E), suggesting that HIF1 $\alpha$ accelerates ID1 degradation in hypoxia through APC/CDH1. This possibility was further supported by results of protein co-IP experiments, in which we found that the ID1 and APC/CDH1 interaction was enhanced in hypoxia in HIF1 $\alpha$-expressing cells but not in HIF1 $\alpha$-KD cells (Figure 2F); in addition, HIF1 $\alpha$ was able to interact with both ID1 and APC/CDH1 in hypoxia, but not with other members of the ID family (Figure 2G). In contrast, HIF $2 \alpha$ was not found to be associated with ID1 (Figure 2H). Thus, it appears that there is a specific negative regulation of ID1 by $\mathrm{HIF} 1 \alpha$ via APC/CDH1.

\section{ID1 Confers Resistance to HIF1 $\alpha$-Targeted Inhibitions}

HIF $1 \alpha$ is a drug target for anticancer therapies (Semenza, 2003). Since ID1 is a well-established oncoprotein that promotes cancer cell survival and proliferation (Perk et al., 2005), we hypothesize that the increase of ID1 may confer adaptive resistance to HIF $1 \alpha$-targeted inhibition. In prostate, liver and brain cancer cell lines, ID1 was inhibited in hypoxia in HIF1 $\alpha$-expressing controls, but was consistently reinstated in HIF $1 \alpha-\mathrm{KD}$ cells (Figure 3A). Stable HIF1 $\alpha$ shRNA knockdown (HIF1-KD) initially inhibited tumor cell survival and growth in hypoxia (P0 in Figure 3B). However, resistance was developed via serial passage (P0-P10 in Figure 3B). In $\mathrm{P} 10$ cells, western blots confirmed that HIF1 $\alpha$ protein remained inhibited, while ID1 was reinstated (Figure 3C). In xenograft experiments, we found that tumors established with the resistant cells (HIF1 $\alpha-\mathrm{KD}-\mathrm{P} 10)$ grew as aggressively as the HIF1 $\alpha$-expressing controls, suggesting that the in vitro hypoxia selected resistance was sufficient to confer resistance in vivo (Figure 3D). On the other hand, tumors by cells sensitive to HIF1 $\alpha-\mathrm{KD}$ (P0) exhibited a slower growth pattern, but grew to similar sizes as the controls (Figures 3E-G), suggesting de novo resistance. After tumors were harvested, we isolated human epithelial tumor cells from the Hep3B xenograft and re-establish them as xenograft-derived subclones (2 clones, Hep3B-xd-c1/2). Western blots showed that, in hypoxia, ID1 was inhibited in Control-xd cells, but was reinstated in HIF1 $\alpha-\mathrm{KD}$-xd cells (* in Figure 3H). Within the ID family (ID1-ID4), ID3 was also inhibited in hypoxia, but not reinstated; ID2 and ID4 were unchanged (Figure $3 \mathbf{H}$ ). Also, other proteins known to confer adaptation to HIF1 inhibitions, e.g., Myc, IL-8, and VEGFA, were unchanged (Figure 3H).

To determine the role of ID1 in conferring the resistance above, we transduced the parental and resistant shHIF $1 \alpha-$ p 10 subclones with lentiviral shRNA silencing ID1 (Figure 4A), and performed colony formation assays in $20 \%$ and $1 \% \mathrm{O}_{2}$. In normoxia, ID1-shRNA alone was growth inhibitory to both 
A
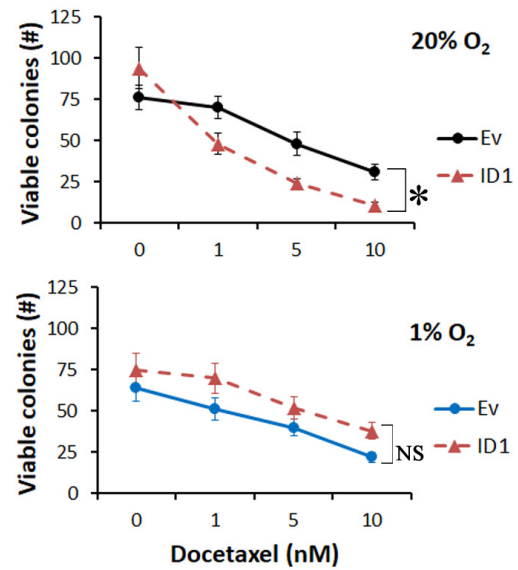

$\mathbf{F}$

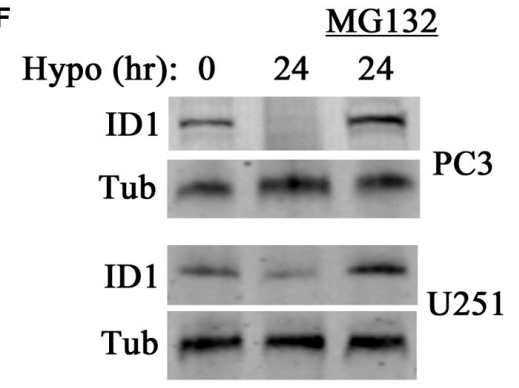

B

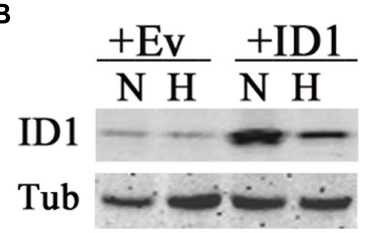

c

C $\quad \frac{\mathrm{Hep} 3 \mathrm{~B}}{\mathrm{NC3}} \frac{\mathrm{U} 251}{\mathrm{NH}}$

ID1

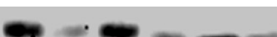

Tub

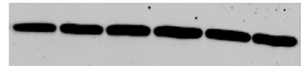

G

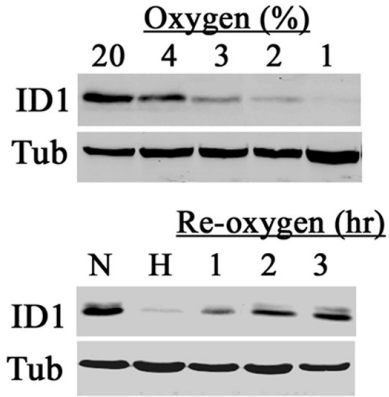

D

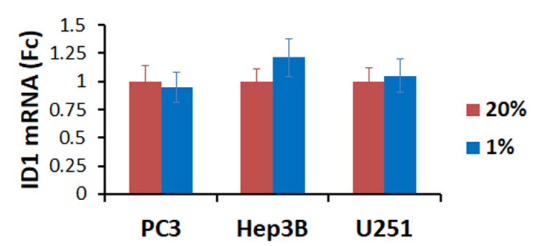

E

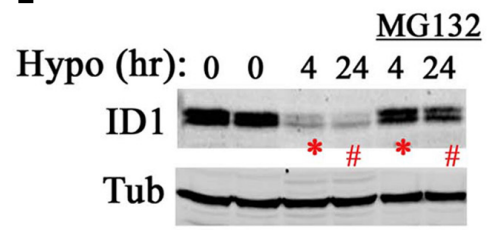

H

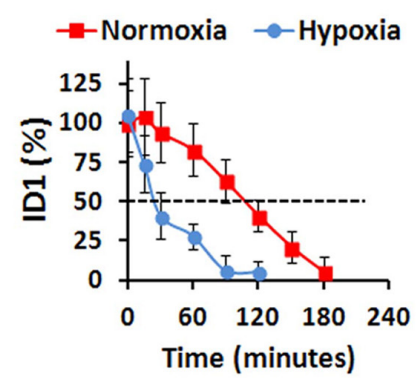

FIGURE 1 | Hypoxia inhibits ID1 proteins. (A) LNCaP cells with stable ID1 (ID1) or empty vector (Ev) overexpression were treated with increasing dose of docetaxel in $20 \%$ or $1 \% \mathrm{O}_{2}$. Cell viability was measured by colony formation assay. ${ }^{*} P<0.05$, $t$-test, $n=3$, mean and standard deviation (stdv). (B) LNCaP-Ev and LNCaP-ID1 cells were cultured in $20 \%(\mathrm{~N})$ or $1 \%(\mathrm{H}) \mathrm{O}_{2}$ for $24 \mathrm{~h}$. ID1 and tubulin (Tub) protein levels were determined by western blots. (C,D) Hep3B, PC3, and U251 cells were cultured in $20 \%(\mathrm{~N})$ or $1 \%(\mathrm{H}) \mathrm{O}_{2}$ for $24 \mathrm{~h}$. ID1 and tubulin were determined by western blots (C). ID1 mRNA levels were determined by qRT-PCR with b-actin as control (D). (E) Hep3B cells were cultured in 1\% $\mathrm{O}_{2}$ for 4 and $24 \mathrm{~h}$ with/without proteasome inhibitor MG132. ID1 and tubulin were measured by western blots. (F) ID1 protein levels in PC3 and U251 cells after 24 h of normoxia, hypoxia, or hypoxia plus MG132. (G) ID1 protein levels after U251 cells were cultured with decreasing concentrations of $\mathrm{O}_{2}$ for $24 \mathrm{~h}$ (top). ID1 proteins after $\mathrm{U} 251$ cells cultured in $20 \%(\mathrm{~N}), 1 \%(\mathrm{H}) \mathrm{O}_{2}$ overnight, or $1 \%$ O overnight followed by re-oxygenation in $20 \% \mathrm{O}_{2}$ for the indicated times (bottom). (H) $\mathrm{U} 251$ cells were cultured in $20 \%$ or $1 \% \mathrm{O}_{2}$, and treated by cycloheximide to arrest protein synthesis. ID1 protein levels at the indicated times were determined by western blots.

cell lines (Figure 4B). In hypoxia, ID1-shRNA had no effect to HIF1 $\alpha$-expressing parental cells, but significantly inhibited the shHIF1 $\alpha$-p10 (Figure 4B). Next, we treated the cells with HIF inhibitor digoxin. In hypoxia, we found that digoxin (100 nM) inhibited the colony formation of HIF $1 \alpha$-expressing cells, but not the resistant shHIF1 $\alpha$-p10 cells (Figure 4C). Importantly, ID1-shRNA was growth-inhibitory in hypoxia against the digoxin-resistant cells (Figure 4C). In xenograft experiments, HIF1 $\alpha$-KD-p10 tumors were resistant to digoxin, but stable ID1-shRNA increased the sensitivity (Figure 4D). On the other hand, ID1-low LNCaP xenografts were sensitive to digoxin, but not in ID1-overexpressing counterpart (Figure 4E).

\section{ID1 Confers HIF1-Independence via Metabolic Compensations}

HIF1 $\alpha$ orchestrates hypoxia response by the upregulation of gene expressions. To further understand the interaction between HIF $1 \alpha$ and ID1, we evaluated the effect of ID 1 on HIF1 $\alpha$ protein level and transcriptional activity. We found that ectopic increase or siRNA silencing of ID1 had no significant effect to HIF1 $\alpha$ protein levels (Figure 5A), but attenuated its transactivation activity (ID1 transfection) (Figure 5B). We next used Affymetrix cDNA microarray to determine the effect of ID1 on hypoxiainduced gene expression (Figure 5C). We found that a subset of hypoxia-upregulated genes was significantly reduced in cells with ID1 overexpression (Figure 5D), among which genes mediating biosynthesis, carbohydrate metabolism, and cell differentiation were significantly enriched (Figure 5E). Mechanistically, we found that ID1 negatively regulates hypoxia-response genes by binding to HIF1 proteins (Figure 5F), and reducing HIF1 recruitments to the target gene promoter, e.g., HK2 (Figure 5G), and gene expression (Figure $\mathbf{5 H}$ ).

On the other hand, ID1 overexpression also increased gene expression. GO-term analysis showed that the increase was enriched at pathways for cell mobility and invasion (Figure 6A), in agreement with previous finding that ID1 promotes tumor cell migration and Matrigel invasion (Lyden et al., 1999). For 


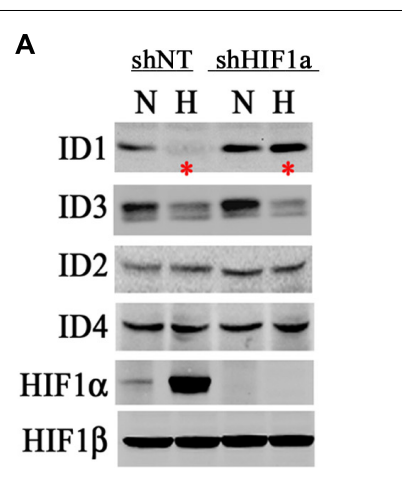

B

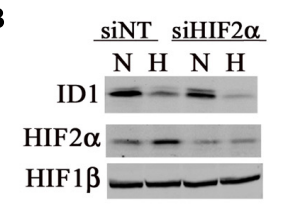

C

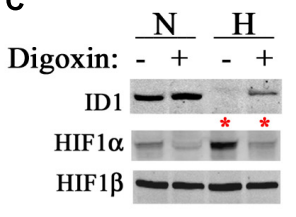

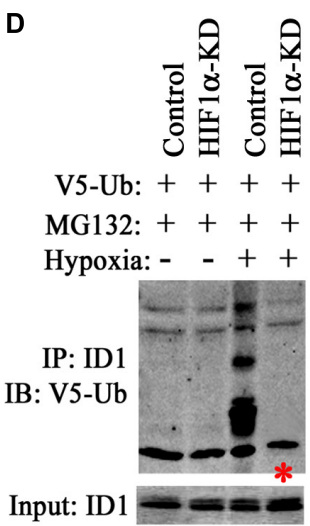

H

G

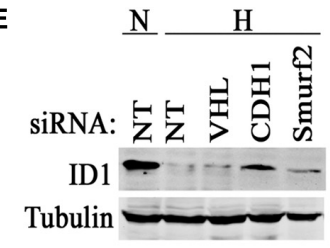

$\mathbf{F}$

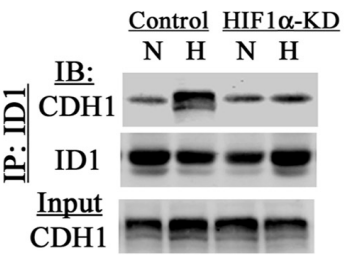

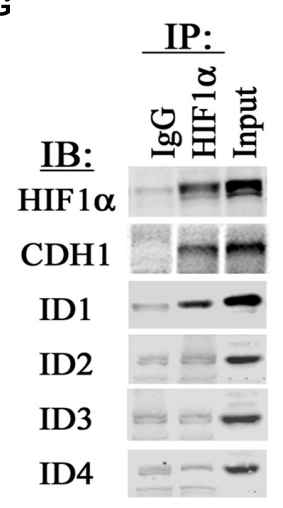

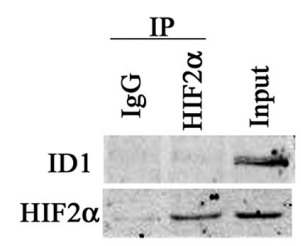

FIGURE 2 | HIF1 $\alpha$ accelerates ID1 protein degradations. (A) Western blots of ID1-ID4, HIF1 $\alpha$, and HIF1 $\beta$ in PC3 cells isogenic for HIF1 $\alpha$ (shNT/shHIF1 $\alpha$ ) in 20\% (N) or $1 \%(H) \mathrm{O}_{2}$. (B) Western blots of ID1, HIF2 $\alpha$, and HIF1 $\beta$ in PC3 cells with siRNA silencing HIF2 $\alpha$ in $20 \%(\mathrm{~N})$ or $1 \%(\mathrm{H}) \mathrm{O}_{2}$. (C) Western blots of ID1, HIF1 $\alpha$, and HIF1 $\beta$ in PC3 cells being treated with HIF inhibitor Digoxin (Sigma) in $20 \%$ or $1 \% \mathrm{O}_{2}$. (D) Hep3B cells with stable shRNA knockdown (KD) of HIF1 $\alpha$ or non-targeting control (Control) were transfected with plasmid coding for V5-tagged ubiquitin. Cells were then treated with MG132 in $20 \%$ or $1 \% \mathrm{O}_{2}$, ID1 protein was immunoprecipitated (IP) and immunoblotted (IB) for V5-ubiquitin modification. (E) Hep3B cells were treated with siRNA against VHL, APC/CDH1 (CDH1), or Smurf2 for $48 \mathrm{~h}$. Afterward, cells were cultured in $20 \%(\mathrm{~N})$ or $1 \%(\mathrm{H}) \mathrm{O}_{2}$ for $6 \mathrm{~h}$ and ID1 levels were measured by western blots. (F) Hep3B Control and HIF1 $\alpha-K D$ cells were cultured in normoxia or hypoxia overnight, and ID1 was IP and IB for the association with APC/CDH1. (G) PC3 cells were cultured in hypoxia with MG132 for $6 \mathrm{~h}$. HIF1 $\alpha$ was then IP and IB for associations with APC/CDH1 and ID1-ID4. (H) PC3 cells were cultured in hypoxia with MG132 for $6 \mathrm{~h}, \mathrm{HIF} 2 \alpha$ was then IP and IB for the association with ID1.

the first time, importantly, we found that gene encoding for glutamine pathway enzyme GLS2 was significantly increased in ID1-overexpressing cells in normoxia and hypoxia (Figure 6B). In response to HIF inhibitor-digoxin, GLS2 was increased in ID1-dependent manner (Figure 6C). Functionally, we found that GLS2 played a role in mediating the ID1-dependent resistance to HIF1 inhibition. Cells that were resistant to digoxin were highly sensitive to siRNA targeting ID1 and GLS2 (Figure 6D). The adaptive response was associated with a switch in energy metabolism. In response to hypoxia, parental cells increased cytosolic glycolysis leading to lactate [the Warburg effect (Dang, 2007)] (Figure 6E). However, cells with stable HIF $1 \alpha-\mathrm{KD}$ did not have such response (\# in Figure 6E). Instead, they exhibited an increase in glutamine metabolism (* in Figure $6 \mathrm{E}$ ), which was sensitive to ID1 or GLS2 siRNA, but not affected by GLS1 siRNA (Figure 6F). To further understand the functional significance, we treated the parental and P10-resistant cells with inhibitors targeting glycolysis and glutamine pathways. We found that the parental tumor cells were more sensitive to glycolysis inhibitors in hypoxia (Figure 6G). As it developed adaptive resistance to $\mathrm{HIF} 1 \alpha-\mathrm{KD}$, the sensitivity to glycolysis inhibitors diminished, while it became dependent to glutamine pathway and thus sensitive to glutamine pathway inhibitors (Figure 6G). Further, the switch of sensitivity to glutamine pathway inhibitors were negated by ID1-shRNA (Figure 6H), confirming the ID1dependency.

\section{DISCUSSION}

Hypoxia is a common feature in solid tumors (Bhandari et al., 2019). The oncogenic role of ID1 in hypoxia is less characterized. By studying the interaction between ID1 and HIF1 $\alpha$, we here present a molecular model in which ID1 and 

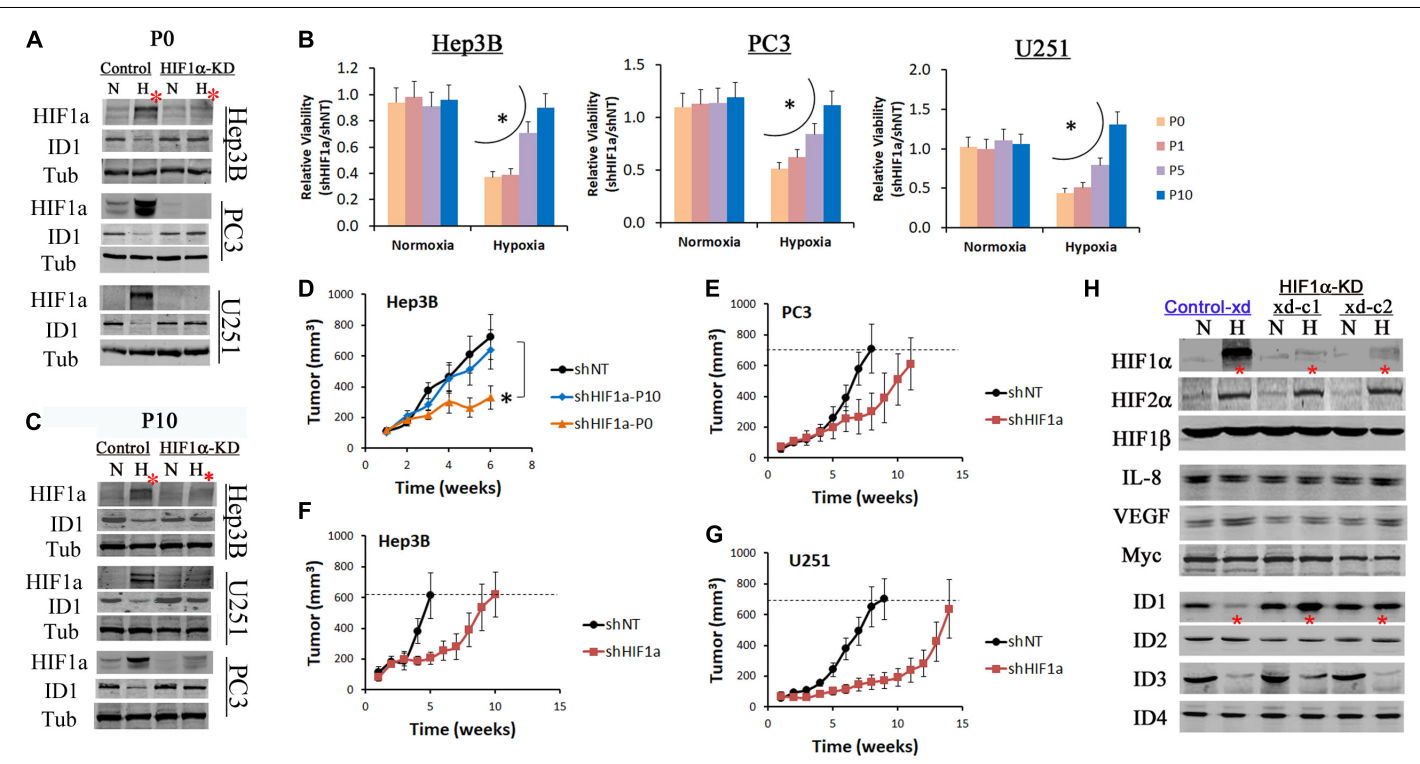

ID4 $--=--$

FIGURE 3 | ID1 is upregulated in tumors resistant to HIF1 $\alpha$ inhibition. Hep3B, PC3, U251 cells were stably transfected by lentiviral shRNA targeting HIF1 $\alpha$ mRNA (HIF1 $\alpha-K D$ ) or non-specific (Control). The resulting cells were cultured in $20 \% \mathrm{O}_{2}(\mathrm{~N})$ or $1 \% \mathrm{O}_{2}(\mathrm{H})$ for $24 \mathrm{~h}$. (A) Western blots of HIF1 $\alpha$ and ID1. Tubulin was used as loading control. The PO indicates cells did not undergo serial passages in hypoxia. (B) Control and HIF1 $\alpha-\mathrm{KD}$ cells from Hep3B, PC3, and U251 underwent serial passages $(1-10)$ in $20 \%$ or $1 \% \mathrm{O}_{2}$. Cell viability was determined by viable cell numbers (shHIF1 $\alpha /$ shNT) after PO, P1, P5 and P10. ${ }^{*} P<0.05$, ANOVA, mean and stdv, $n=3$. (C) Western blots of HIF1 $\alpha$ and ID1 after P10. (D) Control (shNT) and HIF1 $\alpha-K D$ cells (PO and P10) from Hep3B were injected (3 million per injection) into the flank of male nude mice. Tumors were measured by digital caliper, ${ }^{*} P<0.05, t$-test, mean and standard error of mean (SEM), $n=8$. (E-G) Control and HIF1 $\alpha$-KD cells (PO) from PC3, U251, and Hep3B were injected (3 million per injection) into the flank of male nude mice. Tumors were measured by digital caliper, mean and SEM. (H) Hep3B-xd cells from were cultured in $20 \%$ or $1 \% \mathrm{O}_{2}$ overnight. Protein levels were determined by western blot.

\section{A}

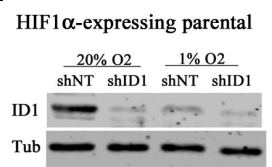

HIF1-KD-p10

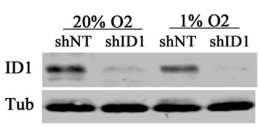

D

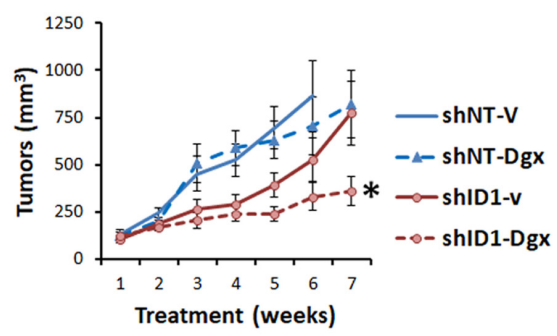

C

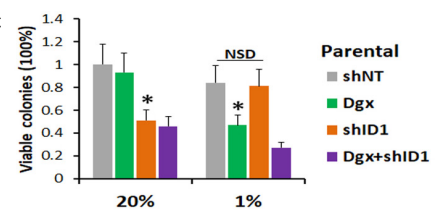

(HIF1+)

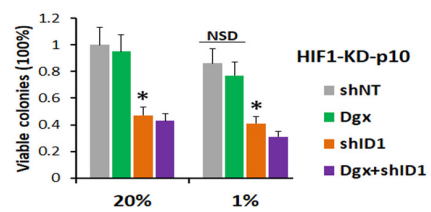

E

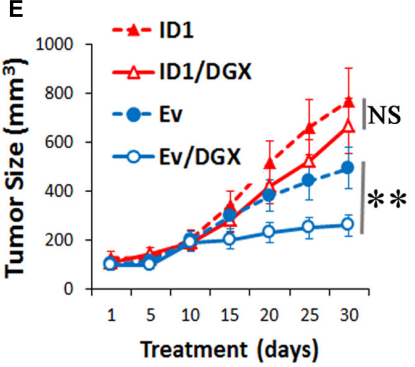

FIGURE 4 | (A) Hep3B-parental and -HIF1 $\alpha$-KD-P10 cells were transduced with lentiviral shRNA-ID1. Cells were cultured in $20 \%$ or $1 \% \mathrm{O}_{2}$ for $24 \mathrm{~h}$, and ID1 and tubulin proteins were determined by western blots. (B) Cells in (A) underwent colony formation assays in $20 \%$ or $1 \% \mathrm{O}_{2},{ }^{*} P<0.05$, $t$-test, mean and stdv, $n=3$. (C) Parental-shNT/shID1 cells or HIF1 $\alpha$-KD-P10-shNT/shID1 cells were treated with HIF inhibitor digoxin and underwent colony formation assays. ${ }^{*} P<0.05, t$-test, mean and stdv, $n=3$. (D) HIF1 $\alpha$-KD-P10-shNT/shID1 cells were injected into the flank of nude mice, and tumor-bearing mice were treated by vehicle or digoxin (Zhang et al., 2008). ${ }^{*} P<0.05$, ANOVA, mean and SEM, $n=6$. (E) LNCaP-Ev/ID1 cells were injected into the flank of male nude mice, and tumor-bearing mice were treated by vehicle or digoxin. ${ }^{* *} P<0.05, t$-test, mean and SEM, $n=6$. 
A

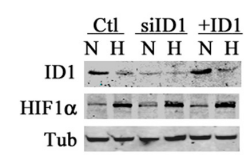

E

\begin{tabular}{lcrc} 
& Fold Enrichment & Raw P value & FDR-P \\
\hline carbohydrate derivative metabolic process & 2.58 & $1.22 \mathrm{E}-05$ & $3.88 \mathrm{E}-02$ \\
regulation of cell differentiation & 2.08 & $2.03 \mathrm{E}-05$ & $4.63 \mathrm{E}-02$ \\
regulation of biosynthetic process & 1.62 & $2.04 \mathrm{E}-05$ & $4.05 \mathrm{E}-02$ \\
regulation of primary metabolic process & 1.5 & $1.43 \mathrm{E}-05$ & $3.79 \mathrm{E}-02$
\end{tabular}

G $\square \mathrm{Ev} \backsim \mathrm{ID1} \backsim \mathrm{Ev}+\mathrm{Hyp} \square \mathrm{ID1}+\mathrm{Hyp}$

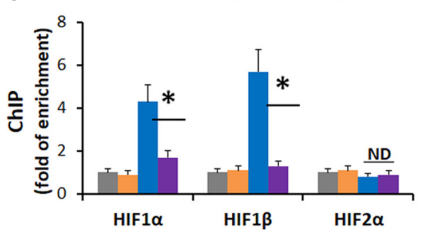

B

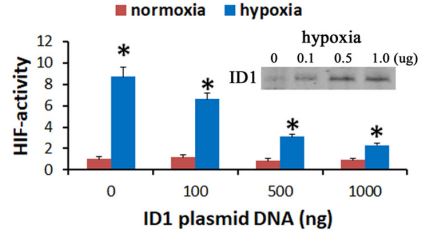

ID1 plasmid DNA (ng)
H
C

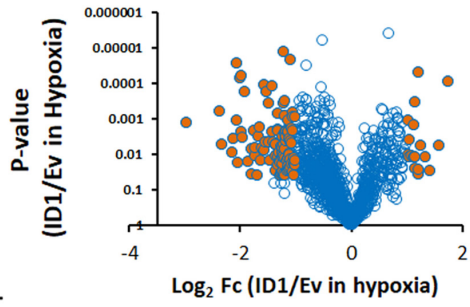

$\mathbf{F}$

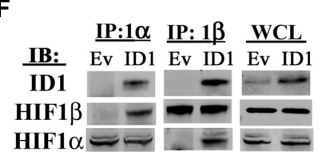

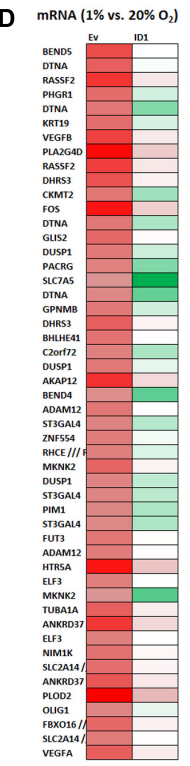

FIGURE 5 | (A) Western blots of ID1, HIF1 $\alpha$, and tubulin (control) in Hep3B cells with siNT/silD1 or Ev/ID1-overexpression in 20\% or 1\% O2. (B) Hep3B-c1 cells that stably transfected with HIF-reporter system (hypoxia/HIF-driving firefly luciferase with constitutive renilla luciferase as control) were transfected with increasing amounts of ID1-overexpressing plasmids, and cultured in $20 \%$ or $1 \% \mathrm{O}_{2}$ for $24 \mathrm{~h}$. HIF-activity was determined by dual-luciferase measurements as described before (Zhang et al., 2008). ${ }^{*} P<0.05$, ANOVA, mean and stdv, $n=3$. Inserts: ID1 western blots. (C) LNCaP-Ev and LNCaP-ID1 cells were cultured in 20\% and 1\% O2 for $24 \mathrm{~h}$, and then underwent Affymetrix cDNA microarray. Volcano plot of hypoxia-response genes in LNCaP-ID1 vs. LNCaP-Ev cells. (D) Heatmap of hypoxia-upregulated genes in Ev-cells, which were attenuated in ID1-cells, color: green $\rightarrow$ red $=$ gene expressions low $\rightarrow$ high. (E) GO pathway analysis of hypoxia-upregulated genes that were attenuated by ID1. (F) Ev or ID1 cells were cultured in $1 \% \mathrm{O}_{2}$ for $24 \mathrm{~h}$. Whole cell lysates (WCL) were immunoprecipitated with antibodies for HIF1 $\alpha$, and probed for ID1, HIF1 $\alpha$, by western blots. (G,H) LNCaP-Ev or -ID1 cells were cultured in $20 \%$ or $1 \%$ O2 for $24 \mathrm{~h}$. The enrichments of HIF1 $\alpha$, HIF1 $\beta$, or HIF2 $\alpha$ (relative to IgG control) at HK2 gene promoter were determined by ChIP (G). The levels of HK2 gene expression were determined by qRT-PCR (H). ${ }^{*} P<0.05, t$-test, mean and stdv, $n=3$.

HIF1 $\alpha$ may drive tumorigenesis in non-hypoxic and hypoxic conditions, respectively. Cancer cells may use this interaction to become more adaptable to the pathologically variable oxygen conditions, thereby gaining survival and growth advantages. In terms of metabolisms, the ID1/HIF1-interaction explains why tumor cells use different energy substrates in non-hypoxic and hypoxic conditions. In non-hypoxic conditions, ID1 supports tumor cells by upregulating GLS2 and glutamine metabolism. In hypoxia, ID1 protein degradation is accelerated, while HIF1 $\alpha$ is activated to support tumors by glycolytic genes and glucose metabolism. In terms of cell cycle regulation, ID1 is a wellestablished driver for cell cycle progression and proliferation in non-hypoxic condition (Sikder et al., 2003). In hypoxia, however, tumor cell may slow down the cell cycle to become more adaptable to the reduced oxygen. Therefore, HIF $1 \alpha$ accelerates the protein degradation of ID1 via APC/CDH1, which is known to coordinate cell cycle progression by protein degradations (Sudo et al., 2001).

Due to the universal occurrence of hypoxia and the intratumor heterogeneity (Movsas et al., 1999; Bristow et al., 2014; Lalonde et al., 2014; Patel et al., 2014), the ID1/HIF1 $\alpha$-interaction may present challenge to HIF-targeted therapies. This may in part explain why HIF-targeted inhibitors have not shown sufficient efficacy in blocking hypoxic tumors. Based on our data, we expect that ID1 is increased in response to HIF-targeted inhibitions, and in turn plays a compensatory role supporting the survival, proliferation, and invasion of tumor cells in hypoxia. Therefore, silencing ID1 may restore or increase tumor sensitivity to HIF1 $\alpha$ inhibition. It also provides a molecular basis for ID1 to be used as a biomarker to predict resistance or efficacy of HIF-inhibitors. In xenograft samples (Figure 3), we detected an increase of ID1 protein level by immunohistochemistry. However, we were unable to clearly define hypoxic regions of the tumor, due to the genetic or chemical inhibition of HIF $1 \alpha$, which we normally use to define hypoxia. Thus, the potential use of ID1 as a marker to predict HIF inhibitor efficacy requires the development of reliable markers of tumor hypoxia, other than HIF1 $\alpha$.

Tumor growth is driven by multiple oncogenic pathways (Hinohara and Polyak, 2019). Our study underscores the importance of understanding the interaction among them. The interaction between ID $1 / \mathrm{HIF} 1 \alpha$ revealed by us suggests a new level of complexity. Clinical tumors are heterogenic in oxygenation. However, most of the genomic analysis of clinical tumors does not differentiate cells based on oxygen. Signals from hypoxic cells can be diluted or masked by non-hypoxic cells. Therefore, the clinical validation of our model requires new approaches focusing on the hypoxic vs. non-hypoxic subsets, e.g., via single-cell analysis. We also speculate that the level of ID1 will be inversely associated with HIF $1 \alpha$ in clinical tumor samples. Many types of tumors acquire HIF $1 \alpha$ in normoxia by oncogenic mutations, thus the negative regulation of ID1 by HIF1 $\alpha$ can occur in normoxia if HIF1 $\alpha$ is available. 
A GO biological process complete regulation of cell motility (GO:2000145) regulation of locomotion (GO:0040012) regulation of cell migration (GO:0030334)

B

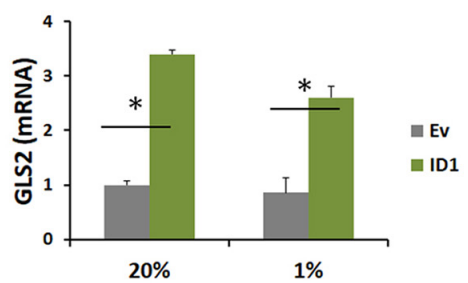

D

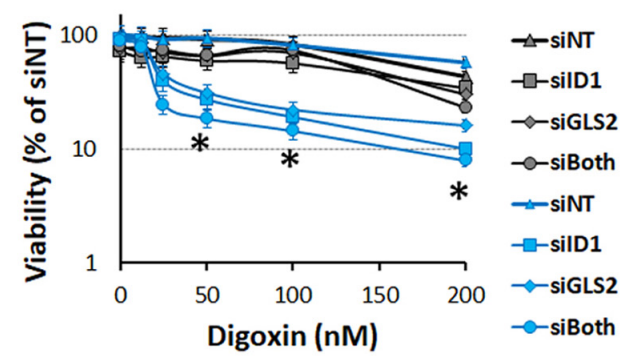

$\mathbf{F}$

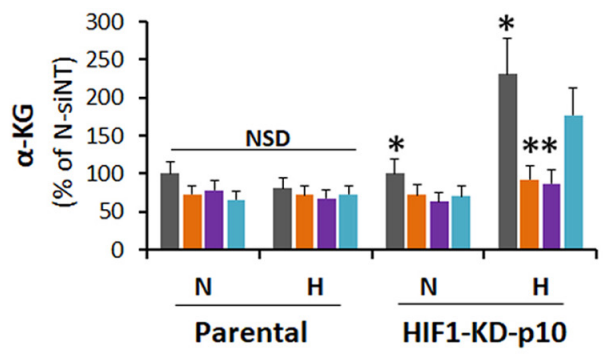

fold Enrichment raw P-value FDR

$\begin{array}{lll}2.77 & 6.22 \mathrm{E}-06 & 2.41 \mathrm{E}-02 \\ 2.64 & 9.08 \mathrm{E}-06 & 2.82 \mathrm{E}-02 \\ 2.73 & 1.91 \mathrm{E}-05 & 4.93 \mathrm{E}-02\end{array}$

$2.73 \quad 1.91 \mathrm{E}-05 \quad 4.93 \mathrm{E}-02$

C

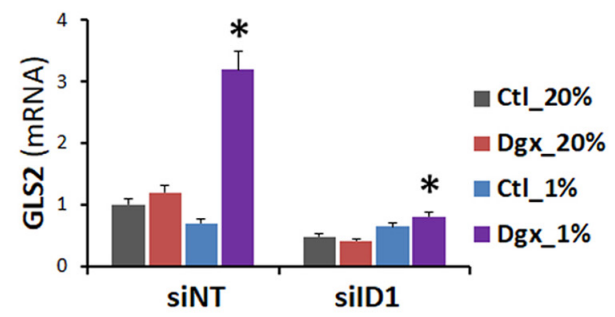

E

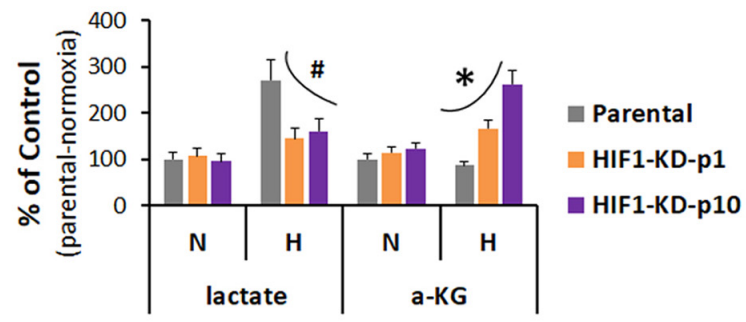

G

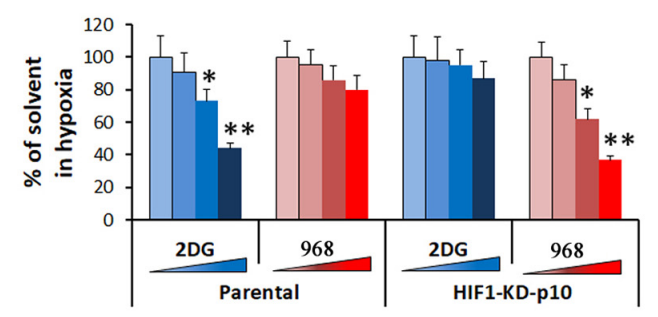

H

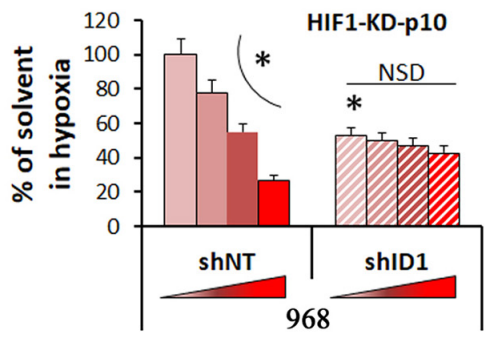

FIGURE 6 | (A) GO pathway analysis of genes upregulated in LNCaP-ID1 vs. LNCaP-Ev cells. (B) LNCaP-ID1/Ev cells were cultured in $20 \%$ or $1 \%$ O 2 , and GLS2 mRNA were determined by cDNA microarray. ${ }^{*} P<0.05$, $t$-test, mean and stdv, $n=3$. (C) Hep3B parental cells were transfected with siRNA (silD1/siNT), and treated by digoxin in $20 \%$ or $1 \%$ O2 overnight. GLS2 were determined by qRT-PCR. ${ }^{*} P<0.05, t$-test, mean and stdv, $n=3$. (D) Hep3B-HIF1-KD-p10 cells were transfected with siRNA (siNT, silD1, siGLS2, or silD1++siGLS2), and treated by increasing concentrations of digoxin in $20 \%$ or $1 \%$ O2 for $72 \mathrm{~h}$. Cell viability were determined by XTT. ${ }^{*} P<0.05$, ANOVA, mean and stdv, $n=3$. (E) Hep3B-parental, -HIF1 $\alpha-K D-P 1$, or -HIF1 $\alpha-K D-P 10$ cells were cultured in $20 \%$ or $1 \% \mathrm{O}_{2}$ for $24 \mathrm{~h}$. Levels of lactate and a-ketoglutarate (aKG) were determined by colorimetric assays from BioVision. $\#,{ }^{*} P<0.05$, ANOVA, mean and stdv, $n=3$. (F) Hep3B-parental/ HIF1 $\alpha$-KD-P10 cells were transfected with siRNA (siNT, silD1, siGLS1, or siGLS2), and cultured in $20 \%$ or $1 \%$ O2 overnight. Then, aKG was determined by colorimetric assays from BioVision. ${ }^{*},{ }^{*} P<0.05$, $t$-test, mean and $\mathrm{stdv}, n=3$. (G) Hep3B-parental/HIF1 $\alpha$-KD-P10 cells were treated with increasing doses of glycolysis inhibitor 2DG or GLS2 inhibitor 968 (Sigma) in $1 \% \mathrm{O}_{2}$. Cell viability was determined by XTT. ${ }^{*},{ }^{* *} P<0.05, t$-test, mean and stdv, $n=3$. (H) HIF1 $\alpha$-KD-p10 cells were transfected with shNT or shID1, and treated by GLS2 inhibitor 968 in $1 \% \mathrm{O}_{2}$ as in (G). Cell viability was determined by XTT, $* P<0.05, t$-test, mean and stdv, $n=3$. 


\section{Experimental Methods and Procedures Cell Culture Conditions}

Prostate (PC3, LNCaP), liver (Hep3B) and brain (U251) cancer cell lines were purchased from ATCC and cultured in RPMI or DMEM media with $10 \%$ FBS and $1 \%$ penicillin streptomycin. The hypoxia or $1 \%$ oxygen condition was created in the cell culture incubator by replacing oxygen with liquid nitrogen. Sodium Bicarbonate $(30 \mathrm{mM})$ was used to neutralize the hypoxia-induced lactate acid for experiments without involving metabolic measurement.

\section{cDNA Microarray}

Gene expression profiles by Affimetrix cDNA microarray were determined at OHSU Gene Profiling Shared Resources as described before (Geng et al., 2018). Each condition had a biological triplicate $(n=3)$. False discovery (FDR) adjusted $t$-test was used to determine the differential expression of individual genes. The level of significance (differentially expressed) was set at $\Delta \log 2$ expression $>1$ or $<-1$ with the FDR-adjust $P$-value $(q)<0.05$. The cDNA microarray data was also analyzed by Gene Set Enrichment Analysis (GSEA), and the enrichment of cancer hallmark pathways was determined with FDR-q.

\section{Gene Knockdown}

As previously described (Geng et al., 2010, 2018; Suwaki et al., 2011), pooled siRNA or lentiviral-based shRNA were purchased from Sigma, and carried out to silence genes, e.g., $\operatorname{HIF} 1 \alpha, \operatorname{HIF} 2 \alpha$, ID1, GLS1, and GLS2. Efficacies of siRNA and shRNA were all determined by western blots.

\section{PCR, Western Blotting, ChIP-PCR}

Quantitative PCR, ChIP-PCR and western blotting were done as previously described (Geng et al., 2018). All RT-PCR primers were purchased from Real Time Primers LLC, and have been verified for human RT-PCR. The antibodies for western blots were purchased from Abcam, BioCheck, R\&D Systems, and Santa Cruz Biotechnology. The ChIP value was adjusted to the IgG as negative control.

\section{Viability/Proliferation/Metabolic Analysis}

Cell viability and proliferation were determined by colony formation assay, XTT, and/or trypan blue exclusion. Cellular lactate and a-ketoglutarate levels were determined by colorimetric kits from BioVision. All these experiments were done as previously described (Geng et al., 2010, 2011, 2018; Liu et al., 2013, 2015).

\section{Xenograft Experiments}

Subcutaneous implants of PC3, Hep3B, U251, and LNCaP cells, including HIF1, ID1 shRNA or overexpressing subclones, were generated in male nude mice as previously described (Geng et al., 2018). The tumor volume was determined by digital caliper measurement, and expressed as \% of growth relative to the start of treatment. All animal experiments are in compliance with protocols approved by OHSU and Johns Hopkins IACUC.

\section{Hypoxia Inducible Factor-Activity Reporter Assays}

As previously described (Zhang et al., 2008), plasmids encoding the firefly luciferase reporter gene under the control of hypoxia response elements or the constitutive renilla luciferase gene (gifts from Dr. Gregg Semenza at the Johns Hopkins University) were co-transfected into the cells, and dual luciferase reporter gene assays were performed with the kit from Promega.

\section{Statistical Analysis}

All experimental data were expressed as mean and standard deviation (SD) unless indicated otherwise. Statistical comparisons between two sample sets were performed with student $t$-test or paired $t$-test, comparisons among more than two samples were performed with repeated measures ANOVA, using MedCalc software. $P<0.05$ was considered as significant, and $P \geq 0.05$ was considered as not significant different (NSD).

\section{DATA AVAILABILITY STATEMENT}

The cDNA microarray data is deposited at Gene Expression Omnibus, GSE185563.

\section{ETHICS STATEMENT}

The animal study was reviewed and approved by the Oregon Health \& Science University and the Johns Hopkins University, Institutional Animal Care and Use Committee.

\section{AUTHOR CONTRIBUTIONS}

DQ and SK designed the study. DQ, SK, and HG analyzed the data and wrote the manuscript. TB, SK, and $\mathrm{H}-\mathrm{KK}$ provided research tools and methods. $\mathrm{HG}, \mathrm{CH}, \mathrm{JP}, \mathrm{CX}, \mathrm{QL}, \mathrm{SW}$, and SK performed the experiments. All authors contributed to the article and approved the submitted version.

\section{FUNDING}

This study was supported by a NIH/NCI grant to DQ, R01CA207377. The Affymetrix cDNA microarray analysis was performed at the OHSU Gene Profiling Shared Resource, supported by the OHSU Knight Cancer Institute NCI Cancer Center Support Grant, P30CA069533. 


\section{REFERENCES}

Alani, R. M., Young, A. Z., and Shifflett, C. B. (2001). Id1 regulation of cellular senescence through transcriptional repression of p16/Ink4a. Proc. Natl. Acad. Sci. U.S.A. 98, 7812-7816. doi: 10.1073/pnas.141235398

Berse, M., Bounpheng, M., Huang, X., Christy, B., Pollmann, C., and Dubiel, W. (2004). Ubiquitin-dependent degradation of Id 1 and Id 3 is mediated by the COP9 signalosome. J. Mol. Biol. 343, 361-370. doi: 10.1016/j.jmb.2004.08.043

Bertout, J. A., Patel, S. A., and Simon, M. C. (2008). The impact of O2 availability on human cancer. Nat. Rev. Cancer 8, 967-975. doi: 10.1038/nrc2540

Bhandari, V., Hoey, C., Liu, L. Y., Lalonde, E., Ray, J., Livingstone, J., et al. (2019). Molecular landmarks of tumor hypoxia across cancer types. Nat. Genet. 51, 308-318. doi: 10.1038/s41588-018-0318-2

Bounpheng, M. A., Dimas, J. J., Dodds, S. G., and Christy, B. A. (1999). Degradation of Id proteins by the ubiquitin-proteasome pathway. FASEB J. 13, 2257-2264. doi: 10.1096/fasebj.13.15.2257

Bristow, R. G., Berlin, A., and Dal Pra, A. (2014). An arranged marriage for precision medicine: hypoxia and genomic assays in localized prostate cancer radiotherapy. Br. J. Radiol. 87:20130753. doi: 10.1259/bjr.20130753

Dang, C. V. (2007). The interplay between MYC and HIF in the warburg effect. Ernst Schering Found. Symp. Proc. 4, 35-53. doi: 10.1007/2789_2008_088

Dang, C. V., Kim, J. W., Gao, P., and Yustein, J. (2008). The interplay between MYC and HIF in cancer. Nat. Rev. Cancer 8, 51-56. doi: 10.1038/nrc2274

Geng, H., Harvey, C. T., Pittsenbarger, J., Liu, Q., Beer, T. M., Xue, C., et al. (2011). HDAC4 protein regulates HIF1 $\alpha$ protein lysine acetylation and cancer cell response to hypoxia. J. Biol. Chem. 286, 38095-38102. doi: 10.1074/jbc. M111.257055

Geng, H., Liu, Q., Xue, C., David, L. L., Beer, T. M., Thomas, G. V., et al. (2012). HIF1 $\alpha$ protein stability is increased by acetylation at lysine 709. J. Biol. Chem. 287, 35496-35505. doi: 10.1074/jbc.M112.400697

Geng, H., Rademacher, B. L., Pittsenbarger, J., Huang, C. Y., Harvey, C. T., Lafortune, M. C., et al. (2010). ID1 enhances docetaxel cytotoxicity in prostate cancer cells through inhibition of p21. Cancer Res. 70, 3239-3248. doi: 10.1158/ 0008-5472.CAN-09-3186

Geng, H., Xue, C., Mendonca, J., Sun, X. X., Liu, Q., Reardon, P. N., et al. (2018). Interplay between hypoxia and androgen controls a metabolic switch conferring resistance to androgen/AR-targeted therapy. Nat. Commun. 9:4972. doi: 10.1038/s41467-018-07411-7

Giaccia, A. J., Simon, M. C., and Johnson, R. (2004). The biology of hypoxia: the role of oxygen sensing in development, normal function, and disease. Genes Dev. 18, 2183-2194. doi: 10.1101/gad.1243304

Giaccia, A., Siim, B. G., and Johnson, R. S. (2003). HIF-1 as a target for drug development. Nat. Rev. Drug Discov. 2, 803-811. doi: 10.1038/nrd 1199

Gupta, G. P., Perk, J., Acharyya, S., de Candia, P., Mittal, V., Todorova-Manova, K., et al. (2007). ID genes mediate tumor reinitiation during breast cancer lung metastasis. Proc. Natl. Acad. Sci. U.S.A. 104, 19506-19511.

Harris, A. L. (2002). Hypoxia-a key regulatory factor in tumour growth. Nat. Rev. Cancer 2, 38-47. doi: 10.1038/nrc704

Hinohara, K., and Polyak, K. (2019). Intratumoral heterogeneity: more than just mutations. Trends Cell Biol. 29, 569-579.

Keith, B., Johnson, R. S., and Simon, M. C. (2012). HIF1 $\alpha$ and HIF2 $\alpha$ : sibling rivalry in hypoxic tumour growth and progression. Nat. Rev. Cancer 12, 9-22. doi: $10.1038 / \mathrm{nrc} 3183$

Kong, Y., Cui, H., and Zhang, H. (2011). Smurf2-mediated ubiquitination and degradation of Id1 regulates p16 expression during senescence. Aging Cell 10, 1038-1046. doi: 10.1111/j.1474-9726.2011.00 746.x

Lalonde, E., Ishkanian, A. S., Sykes, J., Fraser, M., Ross-Adams, H., Erho, N. G., et al. (2014). Tumour genomic and microenvironmental heterogeneity for integrated prediction of 5-year biochemical recurrence of prostate cancer: a retrospective cohort study. Lancet Oncol. 15, 1521-1532.

Lasorella, A., Benezra, R., and Iavarone, A. (2014). The ID proteins: master regulators of cancer stem cells and tumour aggressiveness. Nat. Rev. Cancer 14, 77-91. doi: $10.1038 / \mathrm{nrc} 3638$

Lasorella, A., Stegmüller, J., Guardavaccaro, D., Liu, G., Carro, M. S., Rothschild, G., et al. (2006). Degradation of Id2 by the anaphase-promoting complex couples cell cycle exit and axonal growth. Nature 442, 471-474.
Liu, Q., Geng, H., Xue, C., Beer, T. M., and Qian, D. Z. (2015). Functional regulation of hypoxia inducible factor-1 $\alpha$ by SET9 lysine methyltransferase. Biochim. Biophys. Acta 1853, 881-891. doi: 10.1016/j.bbamcr.2015.01.011

Liu, Q., Harvey, C. T., Geng, H., Xue, C., Chen, V., Beer, T. M., et al. (2013). Malate dehydrogenase 2 confers docetaxel resistance via regulations of JNK signaling and oxidative metabolism. Prostate 73, 1028-1037.

Löfstedt, T., Fredlund, E., Holmquist-Mengelbier, L., Pietras, A., Ovenberger, M., Poellinger, L., et al. (2007). Hypoxia inducible factor- $2 \alpha$ in cancer. Cell Cycle 6, 919-926. doi: 10.4161/cc.6.8.4133

Lyden, D., Young, A. Z., Zagzag, D., Yan, W., Gerald, W., O’Reilly, R., et al. (1999). Id1 and Id3 are required for neurogenesis, angiogenesis and vascularization of tumour xenografts. Nature 401, 670-677. doi: 10.1038/44334

Majmundar, A. J., Wong, W. J., and Simon, M. C. (2010). Hypoxia-inducible factors and the response to hypoxic stress. Mol. Cell 40, 294-309.

Marignol, L., Rivera-Figueroa, K., Lynch, T., and Hollywood, D. (2013). Hypoxia, notch signalling, and prostate cancer. Nat. Rev. Urol. 10, 405-413. doi: 10.1038/ nrurol.2013.110

Movsas, B., Chapman, J. D., Horwitz, E. M., Pinover, W. H., Greenberg, R. E., Hanlon, A. L., et al. (1999). Hypoxic regions exist in human prostate carcinoma. Urology 53, 11-18.

Patel, A. P., Tirosh, I., Trombetta, J. J., Shalek, A. K., Gillespie, S. M., Wakimoto, H., et al. (2014). Single-cell RNA-seq highlights intratumoral heterogeneity in primary glioblastoma. Science 344, 1396-1401. doi: 10.1126/science.125 4257

Perk, J., Iavarone, A., and Benezra, R. (2005). Id family of helix-loop-helix proteins in cancer. Nat. Rev. Cancer 5, 603-614. doi: 10.1038/nrc1673

Powis, G., and Kirkpatrick, L. (2004). Hypoxia inducible factor-1 $\alpha$ as a cancer drug target. Mol. Cancer Ther. 3, 647-654.

Ratcliffe, P. J. (2007). HIF-1 and HIF-2: working alone or together in hypoxia? J. Clin. Invest. 117, 862-865. doi: 10.1172/JCI31750

Semenza, G. L. (2003). Targeting HIF-1 for cancer therapy. Nat. Rev. Cancer 3, 721-732. doi: $10.1038 / \mathrm{nrc1} 187$

Semenza, G. L. (2007). Evaluation of HIF-1 inhibitors as anticancer agents. Drug Discov. Today 12, 853-859. doi: 10.1016/j.drudis.2007.08.006

Semenza, G. L. (2009). HIF-1 inhibitors for cancer therapy: from gene expression to drug discovery. Curr. Pharm. Des. 15, 3839-3843.

Semenza, G. L. (2010). Defining the role of hypoxia-inducible factor 1 in cancer biology and therapeutics. Oncogene 29, 625-634. doi: 10.1038/onc.2009.441

Semenza, G. L. (2012). Hypoxia-inducible factors: mediators of cancer progression and targets for cancer therapy. Trends Pharmacol. Sci. 33, 207-214. doi: 10. 1016/j.tips.2012.01.005

Sikder, H. A., Devlin, M. K., Dunlap, S., Ryu, B., and Alani, R. M. (2003). Id proteins in cell growth and tumorigenesis. Cancer Cell 3, 525-530. doi: 10.1016/S15356108(03)00141-7

Sowter, H. M., Raval, R. R., Moore, J. W., Ratcliffe, P. J., and Harris, A. L. (2003). Predominant role of hypoxia-inducible transcription factor (Hif)-1 $\alpha$ versus Hif- $2 \alpha$ in regulation of the transcriptional response to hypoxia. Cancer Res. 63, $6130-6134$.

Sudo, T., Ota, Y., Kotani, S., Nakao, M., Takami, Y., Takeda, S., et al. (2001). Activation of Cdh1-dependent APC is required for G1 cell cycle arrest and DNA damage-induced G2 checkpoint in vertebrate cells. EMBO J. 20, 6499-6508. doi: 10.1093/emboj/20.22.6499

Sun, X. H., Copeland, N. G., Jenkins, N. A., and Baltimore, D. (1991). Id proteins Id1 and Id2 selectively inhibit DNA binding by one class of helixloop-helix proteins. Mol. Cell Biol. 11, 5603-5611. doi: 10.1128/MCB.11.11. 5603

Suwaki, N., Vanhecke, E., Atkins, K. M., Graf, M., Swabey, K., Huang, P., et al. (2011). A HIF-regulated VHL-PTP1B-Src signaling axis identifies a therapeutic target in renal cell carcinoma. Sci. Transl. Med. 3:85ra47. doi: 10.1126/ scitranslmed.3002004

Trausch-Azar, J. S., Lingbeck, J., Ciechanover, A., and Schwartz, A. L. (2004). Ubiquitin-proteasome-mediated degradation of Id1 is modulated by MyoD. J. Biol. Chem. 279, 32614-32619. doi: 10.1074/jbc.M40379 4200

Wang, G. L., Jiang, B. H., Rue, E. A., and Semenza, G. L. (1995). Hypoxia-inducible factor 1 is a basic-helix-loop-helix-PAS heterodimer regulated by cellular O2 tension. Proc. Natl. Acad. Sci. U.S.A. 92, 5510-5514. doi: 10.1073/pnas.92.12. 5510 
Zhang, H., Qian, D. Z., Tan, Y. S., Lee, K., Gao, P., Ren, Y. R., et al. (2008). Digoxin and other cardiac glycosides inhibit HIF-1 $\alpha$ synthesis and block tumor growth. Proc. Natl. Acad. Sci. U.S.A. 105, 19579-19586. doi: 10.1073/pnas.080976 3105

Conflict of Interest: The authors declare that the research was conducted in the absence of any commercial or financial relationships that could be construed as a potential conflict of interest.

Publisher's Note: All claims expressed in this article are solely those of the authors and do not necessarily represent those of their affiliated organizations, or those of the publisher, the editors and the reviewers. Any product that may be evaluated in this article, or claim that may be made by its manufacturer, is not guaranteed or endorsed by the publisher.

Copyright (๑) 2021 Geng, Ko, Pittsenbarger, Harvey, Xue, Liu, Wiens, Kachhap, Beer and Qian. This is an open-access article distributed under the terms of the Creative Commons Attribution License (CC BY). The use, distribution or reproduction in other forums is permitted, provided the original author(s) and the copyright owner(s) are credited and that the original publication in this journal is cited, in accordance with accepted academic practice. No use, distribution or reproduction is permitted which does not comply with these terms. 